\title{
A Remark on Multistability for Monotone Systems II
}

\author{
German A. Enciso*
}

Abstract-A recent paper by Angeli and Sontag presented a stability criterium for feedback loops involving single input, single output models which admit a well-defined I/O characteristic and satisfy a monotonicity condition. This paper generalizes the argument to arbitrary strongly monotone systems. The main idea is to study the asymptotic behavior of a strongly monotone system by relating it to a graph on the plane which contains information about the equilibria of the system and their stability properties.

\section{INTRODUCTION}

Consider a monotone system

$$
\dot{x}=g(x)
$$

defined on a state space $X \subseteq \mathbb{R}^{n}$ (definitions below). In the paper [2], the asymptotic behavior of such systems is studied by decomposing them as the unity feedback closed loop of a (SISO) controlled monotone system

$$
\dot{x}=f(x, u), u=h(x) .
$$

This system is assumed to have the following property: for every $u_{0} \in U$, the autonomous system $\dot{x}=f\left(x, u_{0}\right)$ with fixed control $u_{0}$ is globally attractive to an equilibrium $k^{X}\left(u_{0}\right)$. The function $k(u)=h\left(k^{X}(u)\right)$ is then used in a graphic test to determine the stability of (1). In the paper [8], Enciso and Sontag generalize this approach to include the multidimensional input case, and they show that the system $\dot{u}=k(u)-u$ has similar stability properties as (1). In the present paper, we eliminate the global attractivity assumption altogether by considering the 'set characteristic' function $K^{X}(u)=\{x \in X \mid f(x, u)=0\}$. We further generalize the results in such a way that no nondegeneracy assumptions are necessary (both of these assumptions were crucial in the previous papers). In order to do so, we make a frequent use of the irreducibility of the system, which means that the digraph $G$ associated to the system (1) is strongly connected, and which is commonly satisfied in nontrivial examples. We have restricted our attention here to the SISO case for reasons of space, but a more comprehensive treatment is given in [10], [11]. The main message of this paper is illustrated in Figure I: since in a strongly monotone system, a generic solution converges to an equilibrium, finding the number of equilibria and their local stability provides an accurate analysis of global behavior, and such information can be read from the graph of the set characteristic.

\footnotetext{
* Mathematical Biosciences Institute, Columbus, OH, USA, email german.enciso@gmail.com. Suported in part by NSF, AFOSR Grant FA9550-04-1-0172, and Dimacs.

** Department of Mathematics, Rutgers University, Piscataway NJ, USA, email sontag@math.rutgers.edu. Suported in part by grants NSF EIA-02-05116 and NSF DMS-0504557.
}

\author{
Eduardo D. Sontag**
}
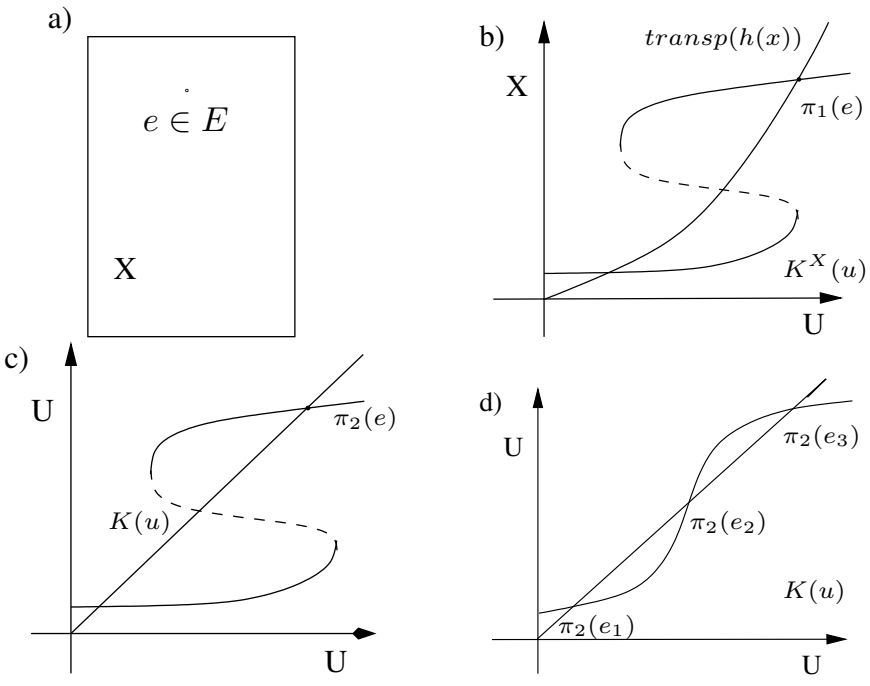

Fig. 1. Interpreting the stability of equilibria in (1) by using the graphs of $K^{X}(u)$ and $K(u)=h \circ K^{X}$. Each equilibrium $e$ in a) corresponds to a point $\left.\pi_{1}(e)=(h(e), e)\right) \in \operatorname{graph} K^{X} \cap \operatorname{transp}(\operatorname{graph} h)$ in b), and to a point $\pi_{2}(p)=(h(e), h(e)$ in $\mathrm{c})$. The first correspondence is always a bijection, whereas the second correspondence is a bijection provided condition $(\mathrm{H})$ is satisfied, see Section III. The point $e$ is guaranteed to be exponentially unstable if $\pi_{i}(e)$ lies on an exponentially unstable branch (see Section IV for definitions), but it may be exponentially unstable even if it is on a stable branch, such as $\pi_{2}\left(e_{2}\right)$ in d). Sufficient and necessary conditions for a hyperbolic point $e$ to be exponentially stable, as will be shown in Section IV, are that both $\pi_{2}(e)$ lies on a stable branch and $K^{\prime}\left(\pi_{2}(e)\right) \leq 1$

The organization of this paper is as follows. After introducing basic definitions and stating some general results in Section II, we describe the relevance of set characteristics in Section III. The main results are presented in Section IV. Section V contains a simple application to a three-variable system, and Section VI considers a cyclic cascade of such systems as a more elaborate example.

The proofs of all lemmas are relatively straightforward, and they have been omitted due to space limitations; see [10], [11] for details.

\section{Definitions}

Let $\mathcal{K} \subseteq \mathbb{R}^{n}$ be a cone, by which is meant a set that is nonempty, convex, closed under multiplication by positive scalars, and pointed (i.e. $\mathcal{K} \cap(-\mathcal{K})=\{0\}$ ).

It will also be assumed that $\mathcal{K}$ is closed and has nonempty interior. The cone $\mathcal{K}$ induces the partial order given by: $x \leq$ $y$ iff $y-x \in \mathcal{K}$, and the stronger order $x \ll y$ iff $y-$ $x \in \operatorname{int} \mathcal{K}$. It will also be said that $x<y$ if $x \leq y$ and $x \neq y$. A commonly used order is that induced by a tuple $s=\left(s_{1}, \ldots s_{n}\right)$, where $s_{i}= \pm 1$ for every $i$, and defined by 
$x \leq_{s} y$ iff $s_{i} x_{i} \leq s_{i} y_{i}$ for every $i$. These cones are referred to as orthant cones.

An autonomous system $\dot{x}=f(x)$ is said to be monotone with respect to $\leq$ if $x \leq y$ implies $x(t) \leq y(t)$ for all $t$, where $x(t), y(t)$ are the solutions of the system with initial conditions $x, y$, respectively. It is strongly monotone if $x<y$ implies $x(t) \ll y(t)$ for all $t>0$. A controlled system (2) is said to be monotone (with respect to given orders in the state and input spaces) if i) the autonomous system $\dot{x}=f\left(x, u_{0}\right)$ is monotone, for each fixed control $u_{0} \in U$, and ii) $u \leq v \rightarrow f(x, u) \leq f(x, v)$ for all $x \in X, u, v \in U$. The function $h$ in this system is said to be a positive feedback function if $x \leq y$ implies $h(x) \leq h(y)$.

A matrix $A \in M_{n \times n}$ is said to be monotone with respect to the order $\leq$ if $x \geq 0$ implies $A x \geq 0$. It is strongly monotone if $x>0$ implies $A x \gg 0$. The matrix $A$ is (strongly) quasimonotone with respect to $\leq$ if the linear system $\dot{x}=A x$ is (strongly) monotone with respect to this order. The leading eigenvalue of $A$, or $\mathrm{s}(A)$, is the eigenvalue with the largest real part among all eigenvalues of $A$. If $A$ is quasimonotone then the Perron-Frobenius theorem guarantees that $\mathrm{s}(A)$ is a real number and that there exists an eigenvector $v>0$ of $A$ associated to $\mathrm{s}(A)$. For a full treatment of this theorem for quasimonotone matrices, see [4] (also [10]), and for the classic statements for monotone systems, see for instance [14].

If the partial derivatives $\partial f_{i} / \partial x_{j}$ have constant sign for every $i \neq j$, one can form the digraph associated to $\dot{x}=f(x)$ by writing a positive (negative) arc from $x_{i}$ to $x_{j}$ iff $\partial f_{i} / \partial x_{j} \not \equiv 0$ and $\partial f_{i} / \partial x_{j} \geq 0\left(\partial f_{i} / \partial x_{j} \leq 0\right)$. A system is monotone with respect to some orthant cone if and only if the digraph of the system has no closed chains with negative parity [3]. The strong monotonicity of a system is closely related with monotonicity together with the strong connectivity of the associated digraph. An important result for strongly monotone systems is Hirsch's generic convergence theorem [13]: almost every bounded solution of a strongly monotone system converges towards the set $E$ of equilibria.

\section{Set Characteristic Functions}

Consider a $C^{1}$ monotone controlled system (2) defined on the input and state spaces $U \subseteq R^{m}, X \subseteq R^{n}$. Assume that $X(U)$ is the closure of an open set in $\mathbb{R}^{n}\left(\mathbb{R}^{m}\right)$, and let $\mathcal{K}_{X} \subseteq \mathbb{R}^{n}, \mathcal{K}_{U} \subseteq \mathbb{R}^{m}$ be the cones with respect to which the system is monotone. Assume that $h$ is a positive feedback function. In this case, the closed loop system

$$
\dot{x}=f(x, h(x))
$$

is easily shown to be monotone, see [2].

Consider the set function $K^{X}: U \rightarrow \mathcal{P}(X)$ defined by $K^{X}(u)=\{x \in X \mid f(x, u)=0\}$. For each fixed $u \in U$, and in the particular case that (2) is strongly monotone, Hirsch's theorem implies that almost all bounded solutions of $\dot{x}=f(x, u)$ converge towards the set $K^{X}(u)$. In this way the present setup generalizes the concept of characteristic proposed in [1], [2], [8], [9]. The idea of generalizing characteristic functions as set characteristics was also used by de Leenheer and Malisoff [7] for a similar setup in the negative feedback case. In the case that $K$ is a single-valued function, and if it holds that i) $\operatorname{det} f_{x}(K(u), u) \neq 0$ for every $u$, and ii) for every fixed point $\bar{u}$ of $K$, $\operatorname{det}\left(K^{\prime}(\bar{u})-I\right) \neq 0$, we say that $K$ is a strong characteristic; this definition corresponds to that of a 'characteristic' in [8].

The equilibria of the closed loop system (3) are in bijective correspondence with the intersection between graph $K^{X}$ and the transpose of graph $h$. We state this in the following lemma, whose proof should be self-evident.

Lemma 1: Given a controlled system (2), a state $x \in X$ is an equilibrium of (3) if and only if $x \in K^{X}(h(x))$. The function $x \rightarrow(h(x), x)$ is a bijective correspondence between equilibria of (3) and points $(u, x)$ such that $x \in$ $K^{X}(u), u=h(x)$.

Given the function $K^{X}$ above, consider the set function $K: U \rightarrow \mathcal{P}(U)$, defined by $K(u)=\left\{h(x) \mid x \in K^{X}(u)\right\}$. The following lemma relates to $K$ as Lemma 1 relates to $K^{X}$. We will say that the system has property $(\mathrm{H})$ if

(H) For every $x_{1}, x_{2} \in E, x_{1} \neq x_{2}$, it holds that $h\left(x_{1}\right) \neq h\left(x_{2}\right)$.

Lemma 2: Let condition $(\mathrm{H})$ be satisfied. Then the function $x \rightarrow h(x)$ forms a bijective correspondence between the equilibria of (3) and the points $u \in U$ such that $u \in K(u)$.

Note that if $K^{X}$ is a strong characteristic, then the assumption of this lemma is satisfied automatically, since $h\left(x_{1}\right)=h\left(x_{2}\right)$ implies $x_{1}=K^{X}\left(h\left(x_{1}\right)\right)=K^{X}\left(h\left(x_{2}\right)\right)=$ $x_{2}$.

Consider now an equilibrium point $\bar{x} \in K^{X}(h(\bar{x}))$ of (3). Let

$$
\dot{x}=A x+B u, y=C x
$$

be the linearization of (2) around $(\bar{u}, \bar{x}), \bar{u}=h(\bar{x})$. Let $k^{X}$ : $S \rightarrow X$ be a $C^{1}$ function defined on an open neighborhood of $\bar{u}$, and such that $f\left(k^{X}(u), u\right)=0$ for all $u \in S$ (such a function is guaranteed to exist if $\operatorname{det} A \neq 0$, via the implicit function theorem). Thus $k^{X}$ can be thought of as a 'branch' of $K^{X}$. A branch is said to be 'stable' if $A(u)$ is Hurwitz for every $u \in S$, and 'unstable' if $A(u)$ is exponentially unstable for every $u \in S$.

From the equation above one can find the derivative $\left(k^{X}\right)^{\prime}(\bar{u})$ by using the chain rule, namely $\left(k^{X}\right)^{\prime}(\bar{u})=$ $-A^{-1} B$. Then the $C^{1}$ function $k: S \rightarrow U$ given by $k(u)=$ $h\left(k^{X}(u)\right)$ is such that $k(\bar{u})=\bar{u}$ and $k^{\prime}(\bar{u})=-C A^{-1} B$. In [10], [11], the stability of the closed loop system is shown to be related with that of the (locally defined) system $\dot{u}=k(u)-u$. Here we will only be concerned with the linearization of that system around $\bar{u}$, namely $\operatorname{Red}(\bar{x}):=$ $-C A^{-1} B-I$. In Theorem 1 we will study the relationship between this matrix and the matrix $A+B C$, which is the linearization of (3) at $\bar{x}$.

\section{The Main Results}

We begin this section with several preliminary lemmas. 
Lemma 3: Let $A$ be strongly quasimonotone with respect to $\mathcal{K}$, and let $B$ be a monotone matrix with respect to $\mathcal{K}$, $B \neq 0$. Then s $(A+B)>\mathrm{s}(A)$.

If $A$ is only assumed to be monotone, then one can still conclude that $\mathrm{s}(A+B) \geq \mathrm{s}(A)$ - see Lemma 24 in [10] or Berman and Plemmons [5].

Lemma 4: Consider matrices $A \in M_{n \times n}, B \in$ $M_{n \times m}, C \in M_{m \times n}$, and assume that $A$ is nonsingular. Then $A+B C$ is nonsingular if and only if $C A^{-1} B+I$ is nonsingular.

The following theorem is a generalization of Theorem 2 in [8].

Theorem 1: Let (4) be a monotone controlled system, and let $A$ be Hurwitz. If $A+B C$ and $-C A^{-1} B-I$ are strongly quasimonotone, then sign $\mathrm{s}(A+B C)=\operatorname{sign} \mathrm{s}\left(-C A^{-1} B-\right.$ $I)$.

Proof: The result in Theorem 2 in [8] covers the case in which det $-C A^{-1} B-I \neq 0$. Therefore it remains only to consider the case $\operatorname{det}-C A^{-1} B-I=0$, or equivalently, $\operatorname{det} A+B C=0$. Define $\lambda:=\mathrm{s}(A+B C)$ and $\mu:=$ $\mathrm{s}\left(-C A^{-1} B-I\right)$, and note that in particular $\lambda \geq 0, \mu \geq 0$, since 0 is in the spectrum of both matrices. We show that $\lambda>0$ if and only if $\mu>0$, which will complete the result.

Suppose first that $\mu>0$. The key observation is that if $\lambda=0$, then there exists a unique vector $\sigma \gg 0$ (modulo multiplication by constant) such that $(A+B C) \sigma=0$, by strong quasimonotonicity and the Perron-Frobenius theorem for quasimonotone matrices. Let $\tau \neq 0$ be such that $-C A^{-1} B \tau-\tau=0$. Multiply by $B$ from the left, and let $w$ be such that $A w=B \tau$. After simplifying, it holds that $-B C w-A w=0$, or $(A+B C) w=0$. Note that $B \tau \neq 0$, since otherwise $0=-C A^{-1} B \tau-\tau=-\tau$. Therefore also $w \neq 0$. If it were true that $\lambda=0$, then by our observation above, $w=\alpha \sigma, \alpha \neq 0$. Now, after multiplying from the left by $C A^{-1}$ and canceling, we get

$$
\left(-I-C A^{-1} B\right) C \sigma=0, C u>0,
$$

which is a contradiction, since by the Perron-Frobenius theorem for q.m. matrices the only eigenvalue of $-I-C A^{-1} B$ that can have positive eigenvectors is $\mu \neq 0$. Thus $\lambda>0$.

Conversely, let $\lambda>0$, and assume by contradiction $\mu=0$. Let $\sigma \gg 0$ be such that $-C A^{-1} B \sigma-\sigma=0$. Let $\tau$ be such that $B \sigma=A \tau$. Note that since $\sigma \gg 0$, it holds that $B \sigma>0$, and therefore $\tau \neq 0$. In the same fashion as above, we have $(A+B C) \tau=0$. Now, since $A$ is quasimonotone and Hurwitz, it holds that for any $x>0$, $A^{-1} x=-\int_{0}^{\infty} e^{t A} x \mathrm{~d} t<0$, see for instance [8], in the proof of Theorem 2. Therefore $\tau=A^{-1} B u<0$. The fact that $-\tau>0$ is an eigenvector of the eigenvalue $0 \neq \lambda$ of $A+B C$ violates the Perron-Frobenius theorem, by strong quasimonotonicity.

For the next theorem we give the following definition. Consider an equilibrium point $\bar{x} \in K^{X}(h(\bar{x}))$ of (3), and the linearization (4) of the system around this equilibrium. We say that $\bar{x}$ is reducible if either $A+B C$ is not strongly quasimonotone or $B C=0$. Otherwise we say that it is nonreducible.
Theorem 2: Consider a $C^{1}$ SISO system (2) which is monotone, and whose closed loop (3) is strongly monotone. Assume that the set of equilibria of (3) is countable. Then almost all bounded solutions of (3) converge towards those equilibrium points $x_{0} \in E$ that are either reducible (if any), or such that $A\left(h\left(x_{0}\right), x_{0}\right)$ is Hurwitz and $-C A^{-1} B-1 \leq 0$.

Proof: As mentioned above, Hirsch's theorem [12] guarantees that almost all states $x \in X$ with bounded orbit satisfy $\omega(x) \subseteq E$. Since any such omega limit set is nonempty and connected, and $E$ is countable, it follows that $w(x)$ is a singleton for almost every $x \in X$ with bounded orbit. That is, almost every bounded solution converges towards some equilibrium point $x_{0} \in E$. Furthermore, By Corollary 4.5 in [13] (which uses the countability of $E$ ), almost every bounded solution converges towards an equilibrium $x_{0} \in E_{s}$.

Consider an equilibrium $x_{0}$ of the closed loop (3), and the linearization (4) of the open loop system around $p=$ $\left(h\left(x_{0}\right), x_{0}\right)$. The linearization of the closed loop around this point has the form

$$
\dot{x}=(A+B C) x
$$

We observe first that if $A(p)$ is exponentially unstable, then $A+B C$ is itself exponentially unstable by the comment after Lemma 3.

Now, let $x_{0}$ be non-reducible and such that $\mathrm{s}(A(p))=0$. We cannot directly apply Lemma 3 , since $A$ is not necessarily strongly quasimonotone. But we know that $A+B C$ is strongly quasimonotone. Therefore there must exist a small number $\epsilon>0$ such that $A+(1-\epsilon) B C$ is strongly quasimonotone (proof: let $T_{\epsilon}$ be the time-1 evolution operator of this matrix. In finite dimensions, it is sufficient to prove that $T$ is strongly monotone for small $\epsilon$. But this is equivalent to $T_{\epsilon}\left(K \cap S^{n}\right) \gg 0$, which is true for small $\epsilon$ by continuity). By the comment after Lemma 3, $0=\mathrm{s}(A) \leq \mathrm{s}(A+(1-\epsilon) B C)$. Since also $\epsilon B C>0$, we apply Lemma 3 to conclude that $\mathrm{s}(A+B C)>0$. Thus this equilibrium is also exponentially unstable. This shows how the exponential stability of $A(p)$ is a necessary condition for a non-reducible point $p$ to satisfy sign $\mathrm{s}(A+B C) \leq 0$.

Finally, let $A(p)$ be a Hurwitz matrix and let $p$ be nonreducible. Since the matrix $-C A^{-1} B-I$ is one-dimensional, and using the assumption that the monotonicity is with respect to orthant cones, we conclude that $\operatorname{signs}(A+B C) \leq$ $0(<0)$ if and only if $-C A^{-1} B-I \leq 0(<0)$. This completes the result.

The assumption in this theorem that the set of equilibria is countable is only made in order to to ensure that the generic solution of the system converges towards an equilibrium (as opposed to towards the set of equilibria). It can be dropped under relatively mild regularity assumptions on the system, such as the differentiability of the vector field and the strong quasimonotonicity of all linearizations around equilibria (see [14] for details). See [10], [11] for several other similar extensions, including generalizing to multiple-input systems. 


\section{An Autoregulatory Transcription Network}

In a study of nitrogen catabolism, Mischaikow et al. [6] consider an eucaryotic unicellular organism (specifically, yeast) which produces a protein that crosses the nuclear membrane and promotes the further production of its own messenger RNA. Let another protein also influence the transcription of the messenger RNA, and denote its (for now fixed) concentration by $\lambda$. Denote by $r, p$ and $q$ the concentrations of the mRNA, the intranuclear protein, and the extranuclear protein respectively, and describe the system by the equations

$$
\begin{aligned}
& \dot{p}=K_{i} q-K_{e} p-a_{2} p \\
& \dot{q}=T(r)-K_{i} q+K_{e} p-a_{3} q \\
& \dot{r}=H(p, \lambda)-a_{1} r .
\end{aligned}
$$

Here the functions $H, T$ are such that $\partial H / \partial p>$ $0, \partial H / \partial \lambda>0, T^{\prime}(r)>0$ and represent the transcription and translation rate, respectively. The constants $a_{1}, a_{2}, a_{3}$ are dilution/degradation coefficients, and the constants $K_{i}, K_{e}$ represent the rates of import and export of protein through the nuclear membrane, respectively. (The original model in [6] uses more arbitrary increasing functions $K_{i}(x), K_{e}(x)$.) In order to study the stability of this model, we write it as the closed loop of the controlled system

$$
\begin{aligned}
& \dot{p}=K_{i} u-K_{e} p-a_{2} p \\
& \dot{q}=T(r)-K_{i} q+K_{e} p-a_{3} q \quad h(p, q, r)=q, \\
& \dot{r}=H(p, \lambda)-a_{1} r .
\end{aligned}
$$

It can be easily verified that this controlled system is monotone, and that for every value of $u$ there exists a unique equilibrium of the system with fixed control $u$. In particular, the variable $q$ converges globally to the value

$$
q(u)=c_{1} T\left(c_{2} H\left(c_{3} u, \lambda\right)\right)+c_{3} c_{4} u,
$$

where $c_{1}=\frac{1}{K_{i}+a_{3}}, c_{2}=\frac{1}{a_{1}}, c_{3}=\frac{K_{i}}{K_{e}+a_{2}}, c_{4}=\frac{K_{e}}{K_{i}+a_{3}}$. The function $q(u)$ corresponds to the set characteristic $k(u)=K(u)$.
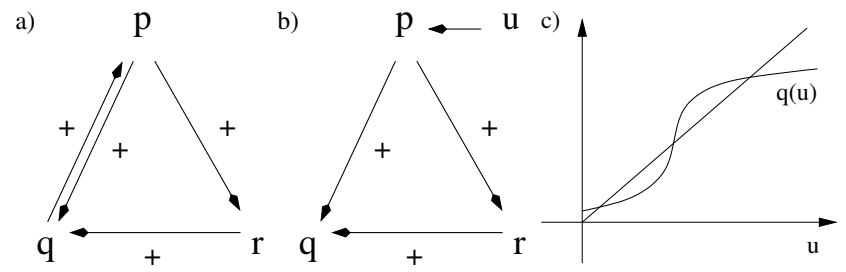

Fig. 2. System (6) has the associated digraph a). Item b) illustrates that of the open loop system (7), and Item c) its associated function $q(u)=$ $K(u)=k(u)$.

We verify that this open loop system contains no reducible points. First, note that the linearization of the closed loop system at any point has the associated digraph in Figure 2 a), since all functions involved have positive derivative. Therefore the matrices $A+B C$ are all strongly quasimonotone. Second, note that the derivative of the right hand side of (7) with respect to $u$ is a nonzero matrix at every point, as is also $\nabla h$. It is easy to verify that the multiplication of these two matrices is nonzero, and the conclusion follows.

Lemma 5: System (6) satisfies property (H).

We conclude by the lemma above that the equilibria of the system are in bijective correspondence with the fixed points of $q(u)$. Furthermore, since there are no reducible equilibria, we can apply Theorem 2, to conclude that almost all (bounded) solutions converge towards the points corresponding to fixed points $\bar{u}$ of $q(u)$ with $q^{\prime}(\bar{u}) \leq 1$.

After studying the stability of system (6) for fixed $\lambda$, we now consider this system itself as a monotone controlled system with control $\lambda$. The previous discussion becomes useful to study the resulting new characteristic function $k^{X}(\lambda)$. Letting now $h(x)=p$ for this new system, one can find the values $p_{\lambda}$ towards which $p$ may converge given a fixed value of $\lambda$. These values form the set characteristic $k(\lambda)$.

The following technical Lemma will be used in the next section. We say that a set function $f$ is injective* if $y \in$ $f\left(x_{1}\right), y \in f\left(x_{2}\right)$ implies $x_{1}=x_{2}$. This is the same as requiring that $f\left(x_{1}\right) \cap f\left(x_{2}\right)=\emptyset$ whenever $x_{1} \neq x_{2}$.

Lemma 6: The function $K(\lambda)$ is injective*.

Most often $K(\lambda)$ consists of two stable branches and one unstable branch, which are joined together as in Figure $3 \mathrm{~d}$ ). In the end of the following section, we will provide a third and final layer of complexity for this case study.
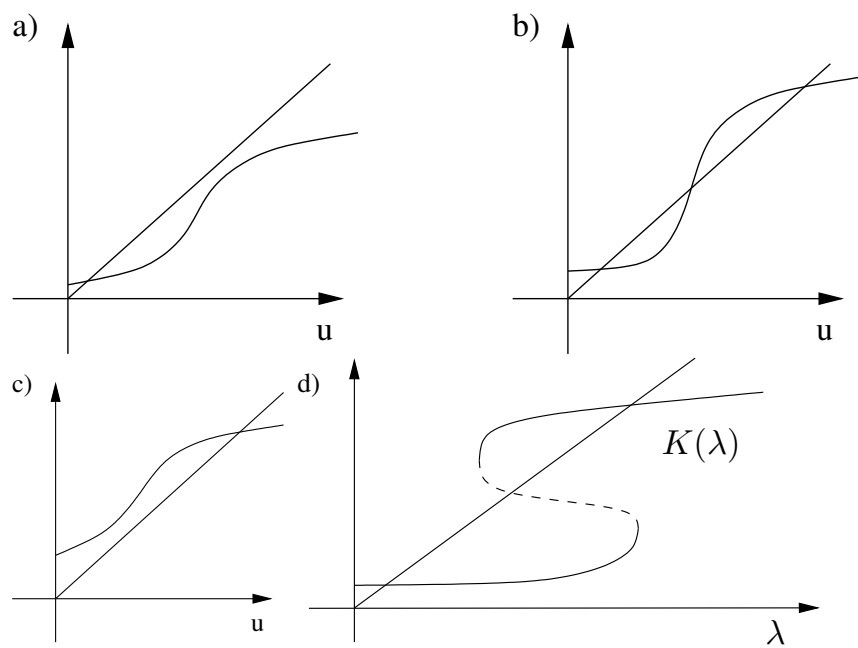

Fig. 3. The function $k(u)$ for a) low, b) medium, and c) high values of the constant $\lambda$. Using the main results, one can conclude which equilibria are Hurwitz for every fixed value of lambda, and therefore draw the function $K(\lambda)$ for the (now) open loop system (6), $h(p, q, r)=q$.

\section{A LARGER EXAMPLE}

Now we are ready for the analysis of a medium scale, monotone dynamical system. Consider a cycle of $k$ proteins $p_{1}, \ldots, p_{k}$, each of which with its respective messenger RNA. Let each protein promote the transcription of its own mRNA, as documented for example in [6] in the case of nitrogen catabolism. Let also each protein $p_{i}$ promote the transcription of $p_{i+1}$, or that of $p_{1}$ in the case of $p_{k}$. The full system therefore looks like 


$$
\begin{aligned}
& \dot{p}_{i}=K_{i m p, i}\left(q_{i}\right)-K_{\text {exp }, i}\left(p_{i}\right)-a_{2 i} p_{i} \\
& \dot{q}_{i}=T\left(r_{i}\right)-K_{i m p, i}\left(q_{i}\right)+K_{\exp , i}\left(p_{i}\right)-a_{3 i} q_{i} i=1 \ldots k \\
& \dot{r}_{i}=H\left(p_{1}, p_{i-1}\right)-a_{1 i} r_{i}
\end{aligned}
$$

where $p_{0}$ is identified with $p_{k}$ throughout. (The model in [6] lets certain inter-protein transcription factors be inhibitory, and doesn't fit the present analysis from here on). Let all constant parameters of this system be positive. As to the nonlinear functions $T_{i}, H_{i}$, assume that they are nonnegative, and that

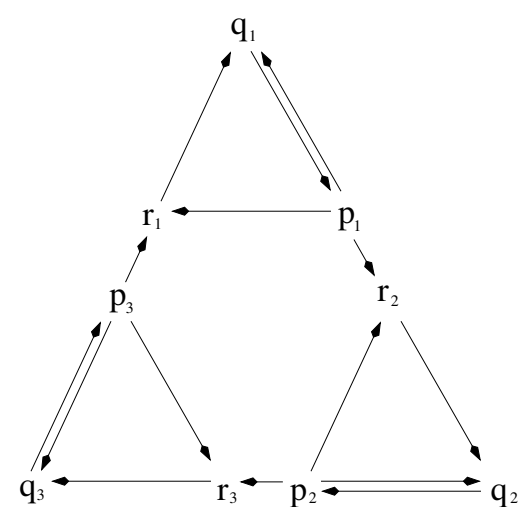

Fig. 4. The digraph associated to system (8) in the case $k=3$.

$$
\begin{aligned}
& T(0)=0, T_{i}^{\prime}(\theta)>0, K_{s, i}(0)=0, \mathcal{K}_{s, i}^{\prime}(\theta)>0, i=1 \ldots k, \\
& s=\text { 'imp', 'exp', } \theta \geq 0 ; \\
& H_{i}(0,0)=H_{i}(0, \phi)=H_{i}(\theta, 0)=0, \nabla H_{i}(\theta, \phi) \gg 0, \\
& i=1 \ldots k, \theta, \phi>0 .
\end{aligned}
$$

See Figure 4 for an illustration. In order to best visualize the behavior of this complex system, only one input will be introduced, and the multi-valued function $K(u)$ will be computed using the tools from digraph decompositions. The open loop system considered is

$$
\begin{aligned}
& \dot{p}_{i}=K_{i m p, i}\left(q_{i}\right)-K_{\exp , i}\left(p_{i}\right)-a_{2 i} p_{i}, i=1 \ldots k, \\
& \dot{q}_{i}=T\left(r_{i}\right)-K_{i m p, i}\left(q_{i}\right)+K_{\exp , i}\left(p_{i}\right)-a_{3 i} q_{i}, i=1 \ldots k, \\
& \dot{r}_{i}=H\left(p_{1}, p_{i-1}\right)-a_{1 i} r_{i}, i=2 \ldots k, \\
& \dot{r}_{1}=H\left(p_{1}, u\right)-a_{1 i} r_{1}, \quad h\left(p_{i}, q_{i}, r_{i}\right)=p_{k} .
\end{aligned}
$$

Lemma 7: The only reducible equilibrium of system (10) is the trivial equilibrium 0 .

The exponential stability of the origin itself can be readily verified by directly computing the Jacobian.

The following is an efficient way to compute the function $K(u)$ as well as its stable branches. The procedure is straightforward, but for a precise setup the reader is referred to [10]. Consider the subdigraph $H_{i}$ of $G$ generated by the nodes $r_{i}, p_{i}, q_{i}$, for each $i=1 \ldots k$. If $K_{i}$ is the characteristic function of the $i$-th system of the form (7), then it follows that $K=K_{k} \circ \ldots \circ K_{1}$ (where the composition is meant in a natural sense).

Lemma 8: System (10) satisfies condition (H).
Theorem 3: Every nonzero equilibrium $e \in E_{s}$ in (8) corresponds bijectively to a non-zero fixed point on a stable branch of the real, multivalued function $K(u)$, with slope less or equal than 1 .

Proof: Follows from the fact that every nonzero equilibrium in $E_{s}$ is non-reducible, Theorem 2, Lemma 2, and Lemma 8.

See [10] for a simple algorithm to write the stable branches of $K$ as a composition of the stable branches for each of the subsystems above.

We implemented these ideas on Matlab, using the functions $H(x, y)=\frac{A_{1} x^{m}+A_{2} y^{n}}{A_{1} x^{m}+A_{2} y^{n}+B_{1}}, T(r)=\frac{A_{4}}{B_{2}+r}, K_{i m p}(q)=$ $K_{i} q, K_{\text {exp }}(p)=K_{e}$.

The function $H$ can be derived using Michaelis-Menten kinetics (quasi steady state analysis) in the case of the gene regulation of two proteins that form $m$ - and $n$-mers before binding to the mRNA protein (resp.) and which complement each other in the sense that either of the two can facilitate the transcription process without the other's help. For simplicity, we will assume $m=4, n=1$ throughout (albeit in the case of cascades of two subsystems, it would be more realistic to let $m=1, n=4$ for the second subsystem, etc). The function $T(r)$ is another Hill-type function with saturation, and the functions $K_{i m p}, K_{\text {exp }}$ are assumed to be linear.
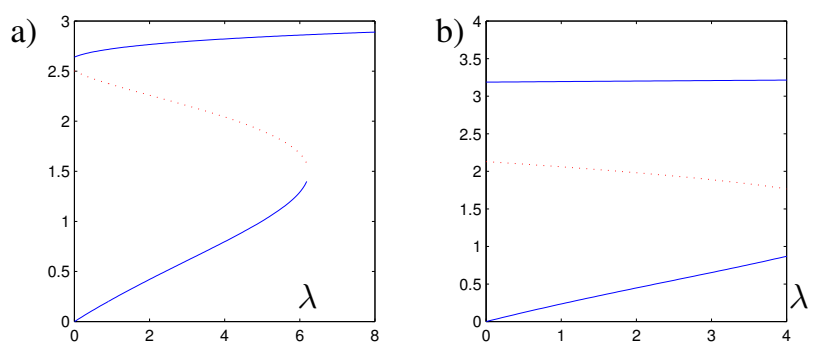

Fig. 5. The multivalued characteristic graphs for system (6), using the parameters sets from (11). Stable branches are solid, and unstable branches are dotted.

c)
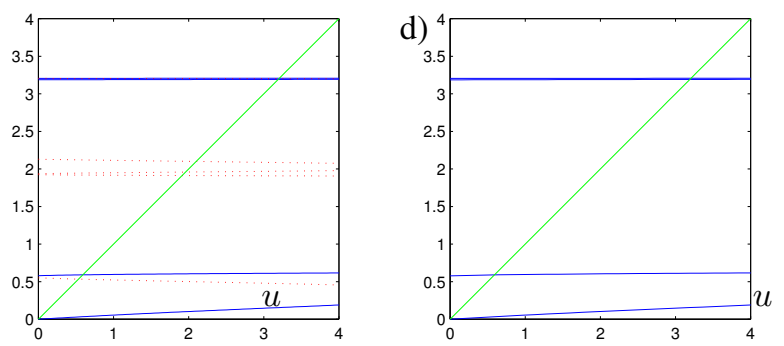

Fig. 6. In chart c), the function $K(u)$ for system (8), $k=2$, using the systems from Figure 5 a) and b) as first and second components, respectively, by composing the functions in these two charts. In chart d), the stable branches are isolated, thus forming the function $S_{H}(u)=S_{H_{2}} \circ S_{H_{1}}$. 
e)

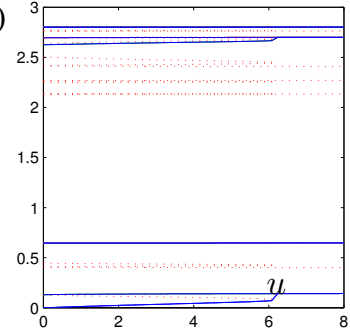

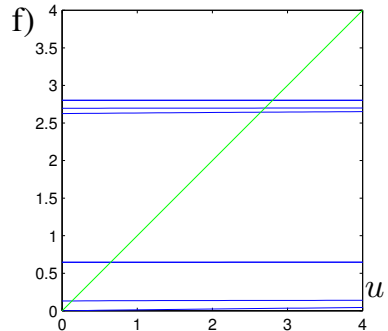

Fig. 7. In e) and f), the same procedure as in Figure 6 is carried out for a system (8), $k=3$, using the systems from Figure $5 \mathrm{a}$ ), b) and a) as first, second and third components, respectively.

Consider two subsystems of the form (6), with parameters

First System: $K_{i}=1 / 6 ; K_{e}=1 / 15 ; a_{1}=1 ; a_{2}=1 / 10$; $a_{3}=1 / 6 ; A_{1}=1 ; A_{2}=1 ; A_{4}=10 ; B_{1}=16 ; B 2=10$, Second System: $K_{i}=1 / 6 ; K_{e}=1 / 12 ; a_{1}=1 ; a_{2}=1 / 12$; $a_{3}=1 / 6 ; A_{1}=1 ; A_{2}=1 ; A_{4}=10 ; B_{1}=16 ; B_{2}=10$,

and coupled in the form (8), $k=2$. Let the system be opened in the form (10), and write its associated digraph as a cascade of the subsystems $H_{1}, H_{2}$ as given above. In Figure $5 \mathrm{a}$ ),b) are illustrated the functions $K(\lambda)$ of the two subsystems in the sense of Section III. Recall that the output of those systems was $h=q$, and that the output of the subsystems here should rather be $p$. To avoid confusion, the constants $K_{i}, K_{e}, a_{2}$ are chosen throughout so that $p=q$ for any equilibrium of a system (6). The characteristic function $K(u)$ of system (8) can therefore be seen as the composition of these two functions, and it is depicted in Figure $6 \mathrm{c}$ ). (Note that the resolution of the graph can present a problem in the upper right corner.) The stable branches of $K(u)$ are the composition of those of the two subsystems - they are illustrated as solid lines throughout the Figure, and separately on Figure $6 \mathrm{~d}$ ).

Every one of the intersections of the graph in Figure $6 \mathrm{c}$ ) with the diagonal represents an equilibrium in system (8). But only a few of those are stable, and they correspond to those points in Figure $6 \mathrm{~d}$ ) on a stable branch (and whose slope is less or equal than one).

In Figure 7, a nine-variable system is considered as in Figure 4. By composing the function in Figure $6 \mathrm{c}$ ) with that in Figure 5 a), the associated set valued characteristic is given in Figure $7 \mathrm{e}$ ), and its associated stable branches in Figure 7 f).

Acknowledgements: We want to thank Patrick de Leenheer for his comments leading to Lemma 1 and Corollary 1, and David Angeli.

\section{REFERENCES}

[1] D. Angeli and E.D. Sontag. Monotone controlled systems. IEEE Trans. Autom. Control, 48:1684-1698, 2003.

[2] D. Angeli and E.D. Sontag. Multistability in monotone input/output systems. Systems and Control Letters, 51:185-202, 2004.

[3] D.L. DeAngelis, W.M. Post, and C.C. Travis CC. Positive Feedback in Natural Systems. Springer-Verlag, New York, 1986.
[4] W. Arendt, A. Grabosch, G. Greiner, U. Groh, H.P. Lotz, U. Moustakas, R. Nagel, F. Neubrander, and U. Schlotterbeck. One-parameter Semigroups of Positive Operators. Springer, 1980.

[5] A. Berman and R.J. Plemmons. Nonnegative Matrices in the Mathematical Sciences. Academic Press, New York, 1979.

[6] E. Boczko, T. Cooper, T. Gedeon, K. Mischaikow, D. Murdock, S. Pratap, and S. Wells. Structure theorems and the dynamics of nitrogen catabolite repression in yeast. Proc. Nat. Acad. Sci, 102:56475652,2005

[7] P. de Leenheer and M. Malisoff. A small gain theorem for monotone systems with multi-valued input-state characteristics. to appear in the IEEE Transactions on Automatic Control, 2005.

[8] G.A. Enciso and E.D. Sontag. Monotone systems under positive feedback: multistability and a reduction theorem. Systems and Control Letters, 7:34-76, 2004.

[9] G.A. Enciso and E.D. Sontag. A remark on multistability for monotone systems. Proceedings of the 43rd IEEE Conference on Decision and Control (CDC), pages 249-254, 2004.

[10] German A. Enciso. Monotone Input/Output Systems, and Applications to Biological Systems. PhD thesis, Rutgers University, 2005.

[11] G.A. Enciso and E.D. Sontag. Multistability for monotone systems under positive feedback. manuscript in preparation, 2005.

[12] M.W. Hirsch. Stability and convergence in strongly monotone dynamical systems. Reine und Angew. Math, 383:1-53, 1988.

[13] M.Hirsch. Systems of differential equations that are competitive or cooperative II: convergence almost everywhere. SIAM J. Math. Anal., 16(3):423-439, 1985.

[14] H.L. Smith. Monotone Dynamical Systems. AMS, Providence, RI, 1995 